\title{
Effect of Light-Emitting Diodes on Cordycepin Production in Submerged Culture of Paecilomyces japonica ${ }^{1}$
}

\author{
Si Young $\mathrm{HA}^{2} \cdot \mathrm{Ji}$ Young $\mathrm{JUNG}^{2} \cdot$ Jae-Kyung YANG $\mathbb{D}^{2, \dagger}$
}

\begin{abstract}
Paecilomyces japonica is widely cultured to produce mycelium for medicinal and health food use. Illumination is an important factor in the growth and production of mycelium in submerged culture. The effects of different light-emitting diode (LED) combinations on the growth and cordycepin content as bioactive substances of mycelium were investigated. The results showed that the mycelium dry weights were lower under dark condition and red LED treatments. Dark condition, fluorescent light, and ultraviolet-A failed to increase the cordycepin content. Blue light was necessary to increase the cordycepin content, and a red-to-blue ratio of 3:7 induced the highest cordycepin content. The cordycepin contents of mycelium in submerged culture were significantly higher in a $12 \mathrm{~h} /$ day illumination time under red and blue (red-to-blue ratio of 3:7) LED treatments, showing an increase of up to $38 \%$ compared with those under the fluorescent-light control condition. The results demonstrated the roles of light with different wavelengths on the biosynthesis of cordycepin as bioactive substances. The low-heat release and replacement of traditional fluorescent lights with low-energy-consuming LEDs could increase the contents of bioactive substances. After optimization of the cordycepin production using response surface methodology (Box-Behnken design) to its canonical form, the optimum combination was found to be as follows: illumination time $=17.7 \mathrm{~h} /$ day, sugar content in the medium $=9.7 \mathrm{~g} / 50 \mathrm{~mL}$, and incubation time $=61.2 \mathrm{~h}$. The model predicted a maximum response of $3779.2 \mu \mathrm{g} / \mathrm{mL}$ cordycepin yield.
\end{abstract}

Keywords: Paecilomyces japonica, cordycepin, light-emitting diodes, submerged culture, response surface methodology

\section{INTRODUCTION}

Paecilomyces japonica (P. japonica) is a valuable nematophagous fungus, which is described in traditional Chinese medicines as rare and exotic medicinal fungi. Some Cordyceps species have long been used for medicinal purposes in China, Japan, Korea, and other oriental countries owing to their various bio- logical and pharmacological properties, which are generally attributed to the presence of important bioactive ingredients such as adenosine, cordycepin, and exopolysaccharides (Kim et al., 2003; Ling et al., 2002; Ng and Wang, 2005). Cordycepin (3-deoxyadenosine), which is a nucleoside analog, is the main bioactive ingredient of Cordyceps and is known to introduce a variety of pharmacological effects (Tuli et al., 2014).

\footnotetext{
${ }^{1}$ Date Received April 1, 2020, Date Accepted June 25, 2020

2 Division of Environmental Forest Science/Institute of Agriculture and Life Science, Gyeongsang National University, Jinju 52828, Republic of Korea

† Corresponding author: Jae-Kyung YANG (e-mail: jkyang68@gmail.com, ORCID: 0000-0003-0423-6398)
} 
Many chemically modified cordycepin derivatives have been reported that have shown various potential therapeutic effects. Cordycepin is reported to inhibit lipopolysaccharide-induced inflammation (Kim et al., 2006) and platelet aggregation (Tuli et al., 2013), exhibit antibacterial (Ahn et al., 2000) and antifungal (Kodama et al., 2000) properties, prevent hyperlipidemia (Guo et al., 2010), induce apoptosis in human neuroblastoma and melanoma cells (Baik et al., 2012), and induce apoptosis and inhibit the proliferation of cancer cells (Tian et al., 2015). Although cordycepin can be chemically synthesized, such a process is cumbersome and requires complicated separation that leads to a low yield and use of a large volume of harmful organic solvents.

Different approaches to cordycepin production from Cordyceps have been reported in previous studies. Shih et al. (2007) reported that the best nitrogen source for cordycepin production was obtained from a submerged culture of Cordyceps militaris (C. militaris) CCRC32219 with yeast extract. Cui and Zhang (2011) proposed a two-stage culture method for cordycepin production, and the highest production levels were obtained in a medium that contained $\mathrm{Mg} 2+$ (Cui and Zhang, 2012). Lin et al. (2012) reported the use of ultraviolet (UV) mutagenesis to derive a mutant strain, namely, Cordyceps SU5-08, which could provide higher cordycepin production. Until recently, however, very low levels of cordycepin have been produced in mycelium and cultured broth under submerged cultivation of Cordyceps sp.. Submerged cultivation of Cordyceps on a laboratory bioreactor scale reported $7.1 \mathrm{mg} / \mathrm{L}$ cordycepin production (Hsu et al., 2002). A submerged-culture method of Cordyceps for cordycepin production on a commercial scale using a two-stage dissolved oxygen control was developed, and moderate cordycepin production of $188.3 \mathrm{mg} / \mathrm{L}$ was realized (Mao and Zhong, 2004). To further enhance the cordycepin production under submerged cul- tivation of Cordyceps, the effects of carbon sources and carbon/nitrogen ratios were investigated using a central composite design and response surface analysis, which resulted in cordycepin production of 345.4 $\pm 8.5 \mu \mathrm{g} / \mathrm{mL}$ (Mao et al., 2005).

Although the aforementioned studies all aimed for high cordycepin production, none focused on the effects of light on the Cordyceps growth and cordycepin production in a submerged culture. Light, which is a type of energy, affects the growth and metabolism of many plants and microalgae (Chen et al., 2010). Danesi et al (2004) reported that the production of biological compounds in Spirulina platensis, such as carotenoid, phycocyanin, and chlorophyll A, were dramatically affected by the use of various light sources. In some cases, light wavelength may also influence fungal growth and metabolites. Some fungi such as Aspergillus nidulans, Aspergillus fumigatus, Gibberrella moniliformis, Ustilago maydis, and Cryptococcuus neoformans were reported to carry photochrome (Ha et al., 2020), which is a photoreceptor that senses red and far-red light (Blumenstein et al., 2005). Dong et al. (2013) discovered that some bioactive components in the fruiting bodies of Cordyceps, such as adenosine, carotenoid, and cordycepin, were significantly enhanced by the regulation of the light wavelength. For instance, light with a short wavelength stimulated the production of carotenoids, thereby suggesting that Cordyceps is a light-sensitive fungus. Thus, its growth and metabolite production may be influenced by illumination conditions (Dong et al., 2012). Although the effect of light on the growth and cordycepin production of Cordyceps fruiting bodies during solid-state cultivation has been observed (Dong et al., 2012), few studies have investigated the growth and cordycepin production of Cordyceps in an illuminated submerged culture. In particular, most of the studies on submerged cultures of Cordyceps have been conducted using C. militaris, and few studies on P. japonica were 
conducted.

In the present study, light-emitting diode (LED) with different wavelengths and their combinations were applied in submerged culture of $P$. japonica to investigate the effects of LED on the mycelium growth and cordycepin content. In addition, the culture conditions were optimized, including the LED illumination time, culture period, and sugar content in a medium, using the response surface methodology (RSM) based on the Box-Behnken design (BBD) and desirability-function analysis to maximize the cordycepin content.

\section{MATERIALS and METHODS}

\subsection{Reagents and standards}

Potato dextrose agar (PDA) and yeast extract were purchased from Himedia Laboratories (Mumbai, India). Glucose and cordycepin were obtained from SigmaAldrich Co. (St. Louis, MO). LED lights and fluorescent lamps were purchased from BISSOL LED Co., Ltd. (Seoul, South Korea), and an incubator were purchased from JEIO TECH Co., Ltd. (SI-900R, Daejeon, South Korea).

\subsection{Mycelium preparation}

P. japonica was obtained from KCCM (Seoul, South Korea). Stock cultures were maintained on PDA plates. Plates were incubated at $25^{\circ} \mathrm{C}$ for 14 days and stored at $4{ }^{\circ} \mathrm{C}$ for use as subcultures every two months (Hidayat et al., 2019). P. japonica was initially grown on PDA medium at $25{ }^{\circ} \mathrm{C}$, and the mycelium harvested after 14 days for experiments.

\subsection{Submerged culture}

Five mycelial agar disks $(5 \mathrm{~mm} \times 5 \mathrm{~mm})$ were obtained using a sterilized punching machine and were transferred to $250-\mathrm{mL}$ flasks containing $100 \mathrm{~mL}$ of yeast extract malt extract glucose (YMG) medium (pH, 6.0; yeast extract, $4 \mathrm{~g} / \mathrm{L}$; malt extract, $10 \mathrm{~g} / \mathrm{L}$; glucose, $4 \mathrm{~g} / \mathrm{L}$ ), PDB medium ( $\mathrm{pH}, 5.2$; glucose, 20 $\mathrm{g} / \mathrm{L}$; potato extract, $4 \mathrm{~g} / \mathrm{L}$ ), and Sabouraud dextrose broth (SDB) medium (pH, 4.5; glucose, $20 \mathrm{~g} / \mathrm{L}$; peptone, $10 \mathrm{~g} / \mathrm{L}$ ) under laminar flow. These three media are different in composition and $\mathrm{pH}$. Using these three media, we intend to evaluate the growth of $P$. japonica mycelium and changes of cordycepin content.

In order to investigate the effect of various amounts of sugar on the production of bioactive compounds of $P$. japonica in the medium, 1.5-15 g of glucose was added per $\mathrm{L}$ to the medium. All incubations were performed five times and these results were used as data for RSM analysis.

Afterwards, the media were applied to submerged culture at $24{ }^{\circ} \mathrm{C}(100 \mathrm{rpm})$ and subjected to different LED light treatments for 7 days.

\subsection{Different wavelengths of LEDs}

To investigate the effects of different wavelengths of LEDs on the cordycepin content of P. japonica, the substrates in the light culture room were exposed to red light LED (619-626 nm), green light LED (526$531 \mathrm{~nm})$, and blue light LED (467-472 nm) for 12 $\mathrm{h} /$ day. The control was illuminated by fluorescent lamps under dark condition, fluorescent lamp or UV-A for 7 days, and all cultivation was performed in five replicates.

To investigate the effects of different illumination time on the content of cordycepin of $P$. japonica, the substrates in the light culture incubator room were illuminated at a light intensity of $1400 \pm 250$ lux for 6,12 , and $24 \mathrm{~h} /$ day by fluorescent lamps, under dark conditions, and UV-A was used as a control. Quintuple samples were exposed to each light treatment for 7 days. 


\subsection{Combination of different ratios of wavelength of LEDs}

Two combinations of different ratios of wavelengths of LEDs were applied for 7 days. These two types were (1) a combination of red and green LED at ratios of $3: 7,5: 5$, and $7: 3,(2)$ a combination of red and blue LED at ratios of $3: 7,5: 5$, and 7:3, and (3) a combination of green and blue LED at ratios of 3:7, 5:5, and 7:3. Cultivation was performed in five replicates.

To investigate the effects of different illumination time on the content of cordycepin of $P$. japonica, the substrates in the light culture incubator room were illuminated at a light intensity of $1400 \pm 250$ lux for 6,12 , and $24 \mathrm{~h}$ /day by fluorescent lamps, under dark conditions, and UV-A was used as a control. Quintuple samples were exposed to each light treatment for 7 days.

\subsection{Characterization and analysis}

\subsubsection{Determination of mycelium dry weight}

Samples collected at various intervals from shake flasks were centrifuged at $6000 \times \mathrm{g}$ for $10 \mathrm{~min}$, and the supernatant was filtered through a pre-weighted Whatman filter paper No. 2 (Whatman International Ltd., Maidstone, UK). The centrifuged mycelium were washed with excess distilled water and collected by filtration through Whatman filter paper; the dry weight of the mycelium was measured after freeze drying to a constant dry weight.

\subsubsection{Determination of cordycepin}

The cordycepin concentration (extracellular product) was analyzed by HPLC. One gram of dry mycelium was extracted with $250 \mathrm{~mL}$ of $50 \%$ ethanol under ultrasonic cleaner for $60 \mathrm{~min}$. The supernatant was separated by centrifugation at $18,400 \times \mathrm{g}$ for $10 \mathrm{~min}$ and then filtered through a 0.45 ìm filter. The filtrate was assayed for cordycepin contents. To measure cor- dycepin, an HPLC system was equipped with a Kinetex 5 ì C18 100A column (Phenomenex, Torrance, CA, USA). The mobile phase was $85 \% \quad 0.02 \quad \mathrm{M}$ $\mathrm{KH}_{2} \mathrm{PO}_{4}$ and $15 \%$ methanol; the flow rate was set at $1.2 \mathrm{~mL} / \mathrm{min}$. The injection volume was $20 \mathrm{iL}$ and the eluent was detected using a UV/Visible Detector (Shimadzu SPA-20A) at $260 \mathrm{~nm}$. Quantification was based on the UV signal response of each standard using the external standard method, and a standard calibration curve $\left(\mathrm{R}^{2}=0.9912174\right)$ was prepared using 100 500 ppm of cordycepin (Sigma-Aldrich Co. (St. Louis, MO)). The calibration curve and HPLC chromatogram of cordycepin standard are shown in Fig. 1.

\subsection{Experimental design by RSM}

A three-level BBD in RSM was employed in the present study, and the optimal conditions were determined through a minimal set of experiments compared with other designs (Dong et al., 2009). BBD was conducted to optimize cordycepin content (Y1) by $P$. japonica. As shown in Table 1, the three factors chosen for this study were light illumination time (h/day, X1), glucose content in submerged media $(\mathrm{g} / 50 \mathrm{~mL}, \mathrm{X} 2)$, and cultivate time (hour, X3) with three levels of each factor: high (coded as +1 ), middle (coded as 0 ), and low (coded as -1 ). For a three-factor, three-level design, the experimental trials were given by a set of points at the midpoint of each edge of a multidimensional cube and three replication of center points, resulting in a total number of 17 experiments. The BBD experimental results were fitted with a second-order polynomial equation (Eq. (1)) by a multiple regression technique:

$$
\mathrm{Y}=\beta_{0}+\sum_{i=1}^{4} \beta_{i} x_{i}+\sum_{i=1}^{4} \beta_{i i} x_{i}^{2}+\sum_{i=1}^{3} \sum_{i<j}^{4} \beta_{i j} x_{i} x_{i j}
$$

where $\mathrm{Y}$ is the predicted response (cordycepin yield in this study, mg/L), $\beta 0, \beta \mathrm{i}, \beta \mathrm{ii}$, and $\beta \mathrm{ij}$ are constant 

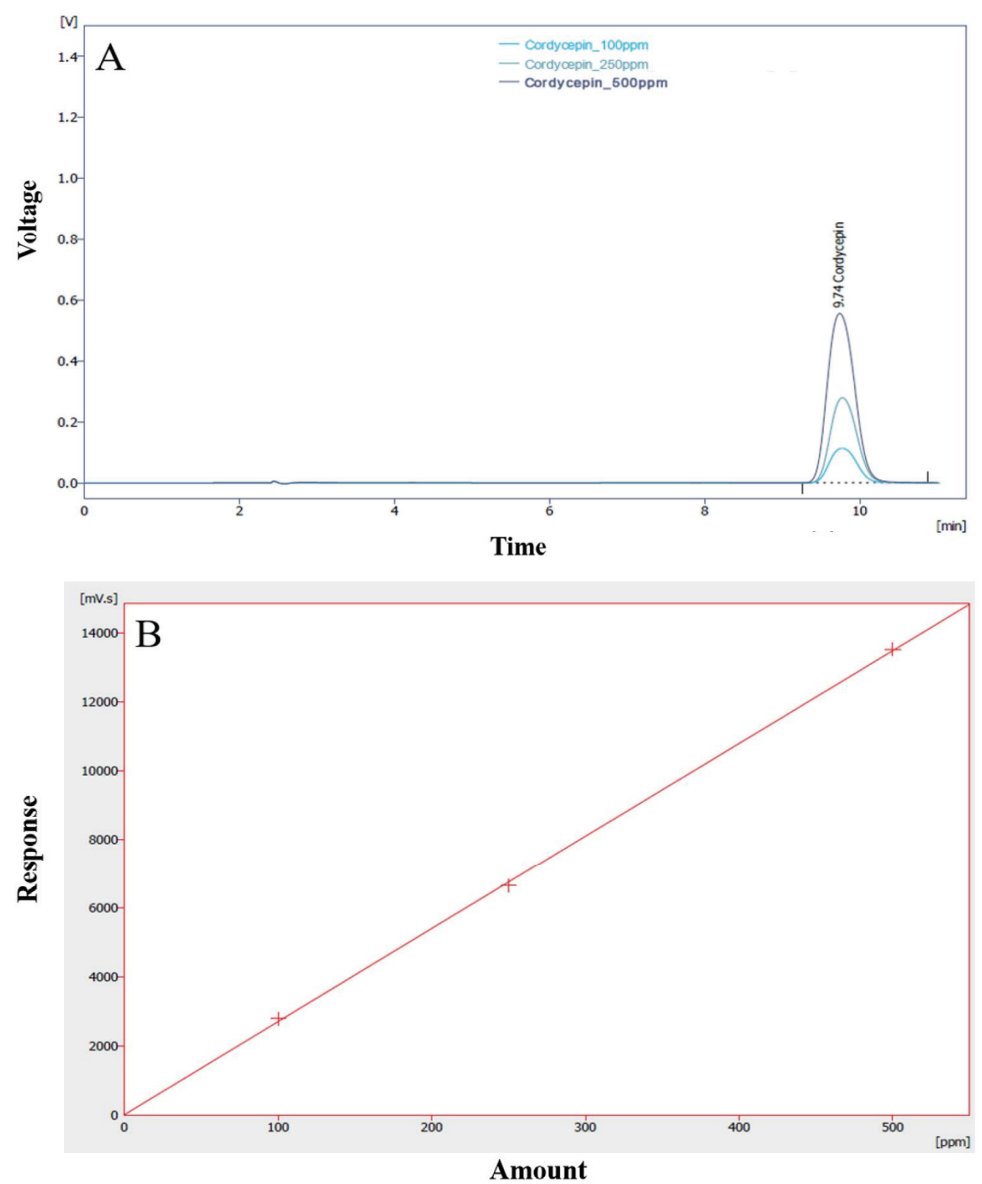

Fig. 1. The HPLC chromatogram and calibration curve of cordycepin standard. A: HPLC chromatogram; B: calibration curve.

coefficients, and $x i$ and $x j$ are the coded independent variables or factors. The quality of fit of the secondorder model equation was expressed by the coefficient of determination $\mathrm{R} 2$, and its statistical significance was determined by an F-test. The significance of the regression coefficients was tested by a t-test. The computer software used was SAS Design Expert 11.

\subsection{Statistical analysis}

Data are presented as the mean standard deviation $(n=3)$. Statistical analyses of the results were performed at $5 \%$ significant level using the Statistical
Analysis System software (SAS institute, Inc., 2000). Differences between the means of individual groups were assessed using SAS with Duncan's multiplerange test.

\section{RESULTS and DISCUSSION}

\subsection{Effect of various media on the mycelium weight and cordycepin content}

Acidic-suitable media components have been reported to be conducive to mycelial growth and pro- 
Effect of Light-Emitting Diodes on Cordycepin Production in Submerged Culture of Paecilomyces japonica

Table 1. Result of three factors, Box-Behnken experimental design

\begin{tabular}{|c|c|c|c|c|c|c|c|}
\hline \multirow[t]{2}{*}{ Run } & \multicolumn{3}{|c|}{$\begin{array}{l}\text { Independent variables } \\
\text { (coded) }\end{array}$} & \multicolumn{3}{|c|}{$\begin{array}{l}\text { Independent variables } \\
\text { (actual) }\end{array}$} & \multirow{2}{*}{$\begin{array}{c}\begin{array}{c}\text { Cordycepin } \\
\text { content, mg/L }\end{array} \\
\text { Y1 }\end{array}$} \\
\hline & $\mathrm{X} 1$ & $\mathrm{X} 2$ & $\mathrm{X} 3$ & $\mathrm{X} 1$ & $\mathrm{X} 2$ & $\mathrm{X} 3$ & \\
\hline 1 & 1 & 0 & -1 & 24 & 7.5 & 24 & 2074.18 \\
\hline 2 & 0 & 0 & 0 & 12 & 7.5 & 72 & 3100.92 \\
\hline 3 & 1 & 0 & 1 & 24 & 7.5 & 120 & 1320.56 \\
\hline 4 & -1 & 1 & 0 & 0 & 10 & 72 & 186.37 \\
\hline 5 & 0 & 0 & 0 & 12 & 7.5 & 72 & 3165.12 \\
\hline 6 & 0 & -1 & -1 & 12 & 5 & 24 & 1772.23 \\
\hline 7 & -1 & 0 & -1 & 0 & 7.5 & 24 & 124.43 \\
\hline 8 & 1 & -1 & 0 & 24 & 5 & 72 & 1913.36 \\
\hline 9 & -1 & 0 & 1 & 0 & 7.5 & 120 & 119.28 \\
\hline 10 & 0 & -1 & 1 & 12 & 5 & 120 & 1112.81 \\
\hline 11 & 1 & 1 & 0 & 24 & 10 & 72 & 3008.74 \\
\hline 12 & 0 & 1 & 1 & 12 & 10 & 120 & 2613.48 \\
\hline 13 & 0 & 0 & 0 & 12 & 7.5 & 72 & 3031.83 \\
\hline 14 & 0 & 0 & 0 & 12 & 7.5 & 72 & 3112.59 \\
\hline 15 & -1 & -1 & 0 & 0 & 5 & 72 & 93.16 \\
\hline 16 & 0 & 1 & -1 & 12 & 10 & 24 & 3655.11 \\
\hline 17 & 0 & 0 & 0 & 12 & 7.5 & 72 & 3712.73 \\
\hline \multirow{2}{*}{\multicolumn{4}{|c|}{ Independent variables }} & \multicolumn{4}{|c|}{ Levels } \\
\hline & & & & -1 & & & 1 \\
\hline \multicolumn{4}{|c|}{$\mathrm{X} 1$ : Illumination time, $\mathrm{h} /$ day } & \multicolumn{2}{|c|}{0} & 12 & 24 \\
\hline \multicolumn{4}{|c|}{$\mathrm{X} 2$ : Sugar content, $\mathrm{g} / 50 \mathrm{~mL}$ medium } & 5 & \multicolumn{2}{|c|}{7.5} & 10 \\
\hline \multicolumn{4}{|c|}{ X3: Incubate time, $\mathrm{h}$} & 24 & \multicolumn{2}{|c|}{72} & 120 \\
\hline
\end{tabular}

duction of metabolites in many types of ascomycetes and basidiomycetes, including Cordyceps sp. (Liu et al., 2011; Leung et al., 2006). The effects of submerged media on the mycelium dry weight and cordycepin content of $P$. japonica are shown in Fig. 2. We can observe that the maximum mycelial dry weights of the yeast malt glucose (YMG) $(\mathrm{pH}$ 6), potato dextrose broth (PDB) (pH 5.2), and sabouraud dextrose broth (SDB)(pH 4.5) media obtained on the seventh day were $8.3,10.2$, and $4.2 \mathrm{~g} / \mathrm{L}$, respectively. The PDB medium is the most commonly used medium in mushroom mycelial culture (Imtiaj and Lee, 2007; Souilem et al., 2017). The cordycepin content of $P$. japonica slowly initiated after the start of the cell growth and reached maximum values of $0.6,1.0$, and $124.8 \mathrm{ig} / \mathrm{mL}$ in the YMG, PDB, and SDB media, respectively. The SDB medium featured a higher glucose content and lower $\mathrm{pH}$ than the others.

This study also confirmed that the cordycepin content varied according to the medium. We need to note that a direct comparison of the metabolite production of different Cordyceps strains from various studies in the literature is difficult because the employed nutrient components and culture conditions were not exactly the same. We selected the SDB medium with the highest cordycepin content of $P$. japonica and proceeded to the next experiments. 


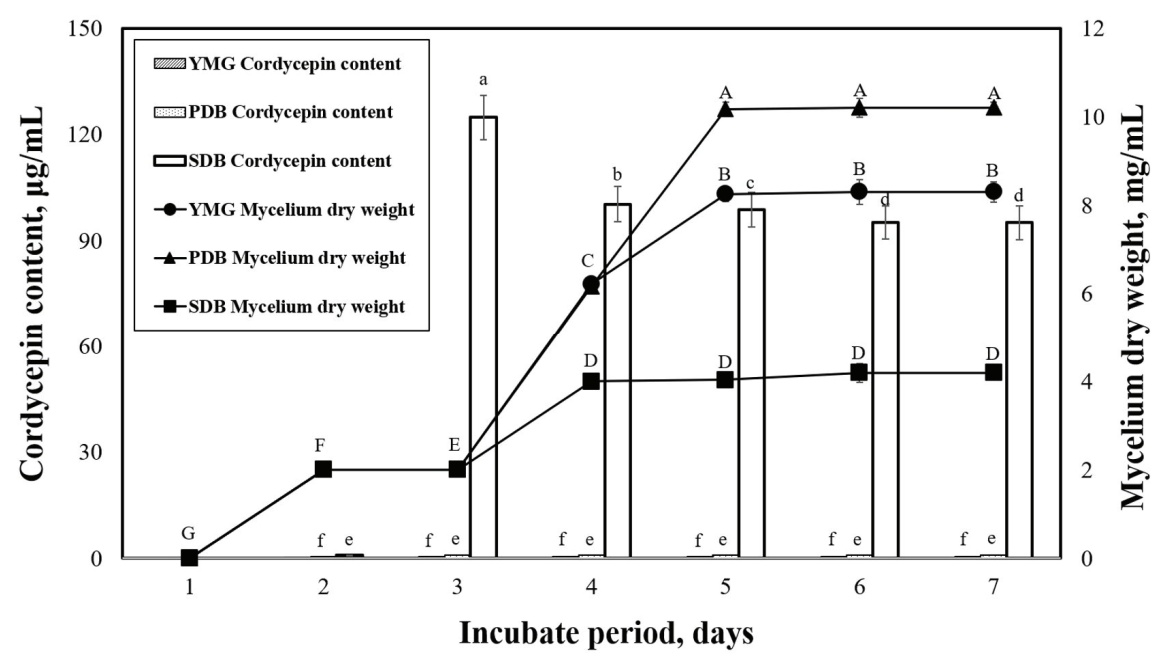

Fig. 2. The effects of various media on the maximal cell growth and cordycepin content by P. japonica in submerged culture. YMG: Yeast extract Malt extract Glucose; PDB: Potato Dextrose Broth; SDB: Sabouraud Dextrose Broth. Each value is expressed as mean $\pm \operatorname{SE}(n=5)$. Different letters on the top of the line represent statistically significant at $5 \%$ probability level (Upper case: mycelium dry weight; small letter: cordycepin content).

\subsection{Effect of LEDs on mycelium dry weight and cordycepin content}

LEDs with different wavelengths were applied on $P$. japonica in a submerged culture to observe the mycelial dry weight (Fig. 3). According to our data, green light provided the best mycelial dry weight of 11.9 $\mathrm{mg} / \mathrm{mL}$. However, red and blue lights led to lower mycelial dry weights of 9.0 and $8.6 \mathrm{mg} / \mathrm{mL}$, respectively, which were very close to the results under dark conditions. When compared with the effects of light on other mushrooms during solid-state cultivation, our study revealed trends similar to previous studies (Cheng et al., 2012; Wu et al., 2013). Cheng et al. (2012) reported that green light enhanced the growth of Aspergillus ficuum, and blue light inhibited fungal growth in a solid-state culture. Various light sources can affect the mycelial growth and cordycepin content in submerged cultivation of $P$. japonica. Therefore, to better understand the effects of light, several experi- ments were carried out in a submerged culture illuminated with LEDs. The effects of LEDs on the cordycepin content of $P$. japonica in submerged cultivation are shown in Fig. 2. Compared with the use of LEDs (green and blue), the control conditions (dark condition, fluorescent light, and UV-A) led to a relatively lower cordycepin content. This result is consistent with what was previously suggested that most Cordyceps strains prefer specific LEDs light color for their growth in submerged cultures (Chiang et al., 2017). The highest cordycepin content (1437.6 ìg/mL) was obtained when blue light was used. The effect of LED on the cordycepin content in a submerged culture has rarely been studied although a previous report has indicated that blue light is a good LED source for cordycepin content from a strain of Cordyceps under solid-state cultivation (Chiang et al., 2017).

Fig. 4 shows the dry-weight values of $P$. japonica mycelia produced under various illumination times when grown under blue LED. The highest mycelia dry 
Effect of Light-Emitting Diodes on Cordycepin Production in Submerged Culture of Paecilomyces japonica

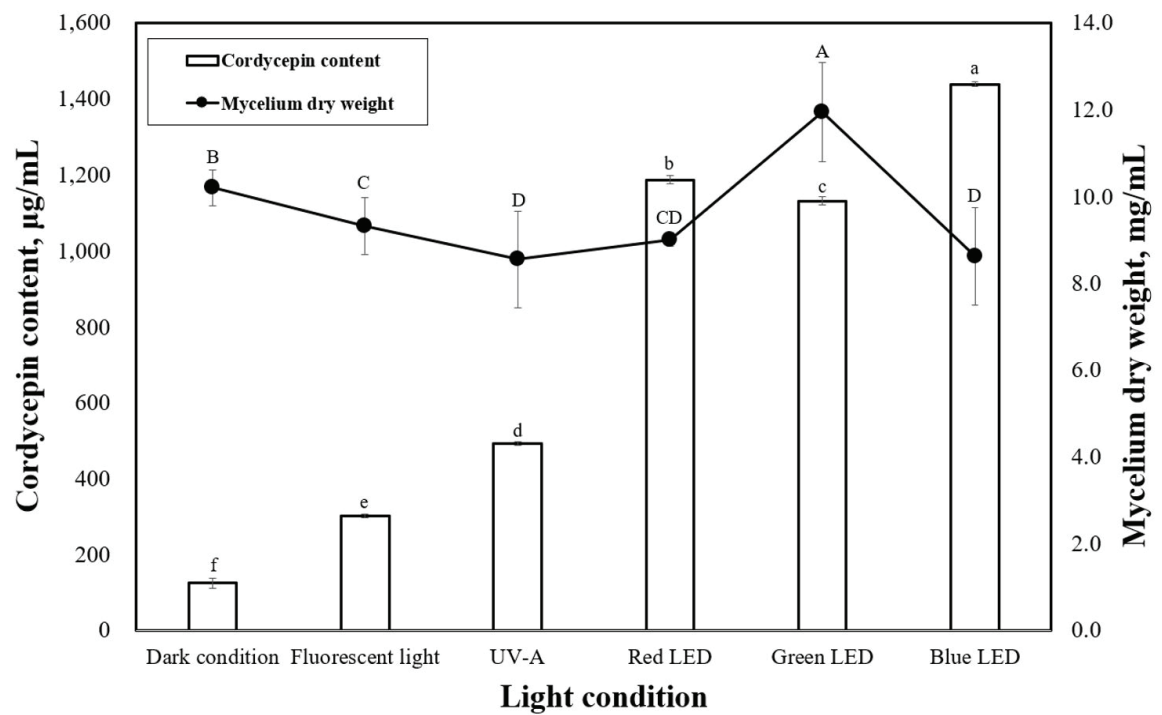

Fig. 3. Effect of LEDs on mycelia dry weight and cordycepin content in submerged culture of $P$. japonica. $P$. japonica was grown using submerged culture in SDB media under $12 \mathrm{~h} /$ day different wavelength LEDs for 3 days. Each value is expressed as mean $\pm \mathrm{SE}$ $(n=5)$. Different letters on the top of the bars represent statistically significant at $5 \%$ probability level (Upper case: mycelium dry weight; small letter: cordycepin content).

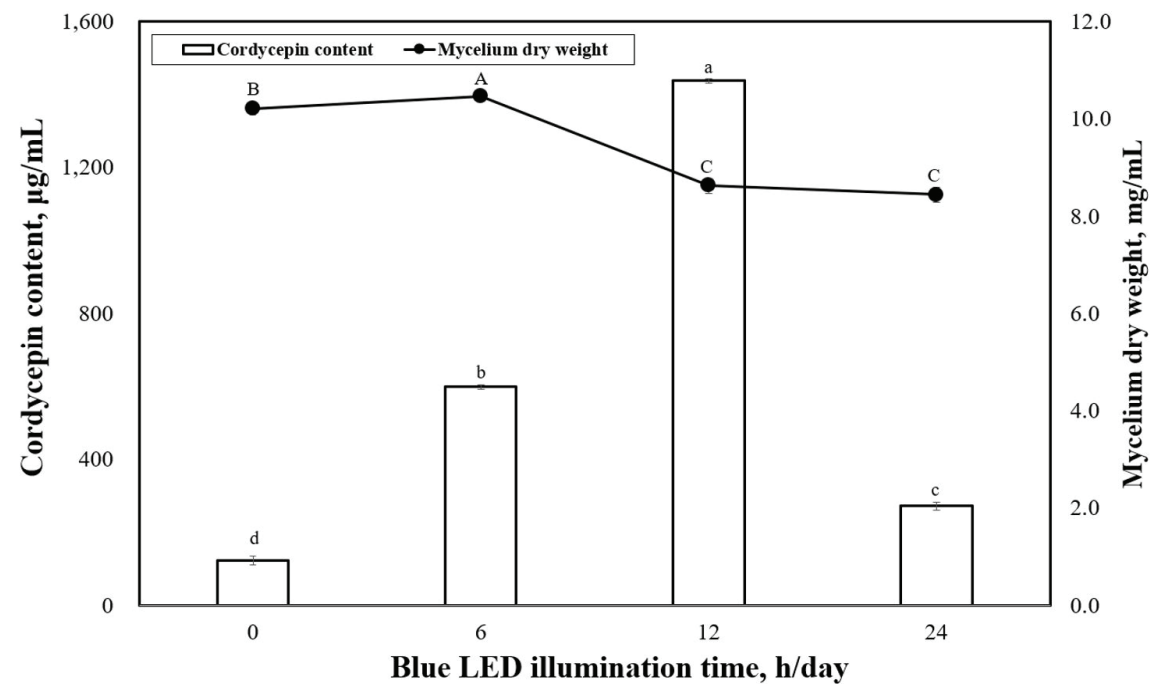

Fig. 4. Effect of illumination time on mycelia dry weight and cordycepin content in submerged culture of $P$. japonica. $P$. japonica was grown using submerged culture in $\mathrm{SDB}$ media under blue LED for 3 days. Each value is expressed as mean $\pm \mathrm{SE}(\mathrm{n}=5)$. Different letters on the top of the bars represent statistically significant at $5 \%$ probability level (Upper case: mycelium dry weight; small letter: cordycepin content). 

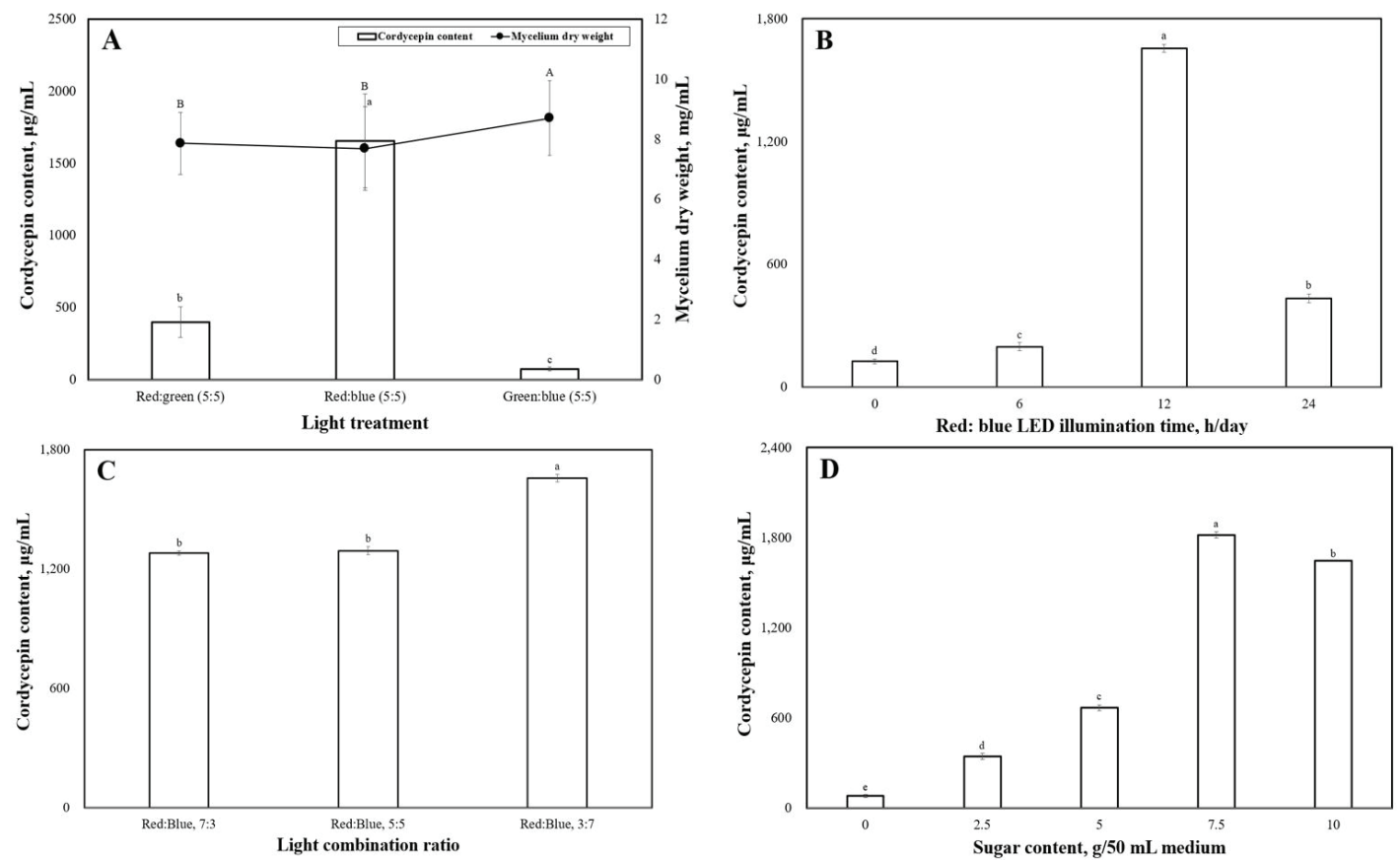

Fig. 5. Effect of LEDs combination or sugar content in medium on mycelia dry weight and cordycepin content in submerged culture of P. japonica. A: P. japonica was grown using submerged culture in SDB media under 12h/day different LEDs combination for 3 days; B: Effect of red:blue illumination time on cordycepin content in submerged culture of P. japonica. P. japonica was grown using submerged culture in SDB under red:blue (5:5) LED combination for 3 days; C: Effect of LEDs combination ratio on cordycepin content in submerged culture of $P$. japonica; D: Effect of sugar content on cordycepin content in submerged culture of $P$. japonica. Each value is expressed as mean $\pm \mathrm{SE}(\mathrm{n}=5)$. Different letters on the top of the bars represent statistically significant at $5 \%$ probability level (Upper case: mycelium dry weight; small letter: cordycepin content).

weight was observed under the 6-h/day illumination time at $10.5 \mathrm{mg} / \mathrm{mL}$, which was significantly different from the 0 -h/day $(10.2 \mathrm{mg} / \mathrm{mL})$. Fig. 4 shows the levels of cordycepin content observed in P. japonica grown under various illumination times using blue LED. The highest cordycepin contents were observed under $12 \mathrm{~h} /$ day $(1437.6 \mu \mathrm{g} / \mathrm{mL})$ of illumination time, whereas the 0 -h/day $(124.8 \mu \mathrm{g} / \mathrm{mL})$ illumination time resulted in the lowest cordycepin concentration. A significant difference was observed in the cordycepin concentrations produced between $0 \mathrm{~h} /$ day (dark condition) and $24 \mathrm{~h} /$ day of illumination time.

\subsection{Effects of LED combination on the mycelia dry weight and cordycepin content}

Fig. 5-A shows the dry-weight value of $P$. japonica mycelia produced under different LED wavelengths and LED-wavelength combinations. Under different LED wavelengths, the highest mycelium dry weight was observed under a combination of green and blue lights, which corresponded to a mycelium dry weight of $8.7 \mathrm{mg} / \mathrm{mL}$, although this was significantly lower than that under the green-light condition (Fig. 2, 11.9 $\mathrm{mg} / \mathrm{mL}$ ). Fig. 5-A shows the cordycepin concentrations under different LED-wavelength combinations. 
The highest cordycepin content was observed under blue light, followed by green light, UV-A, and fluorescent light. Clearly, the production of cordycepin content was optimal with the LED wavelength (Fig. 3), but it was not optimized under any LED-wavelength combination. Thus, achieving an optimal LEDwavelength combination for the production of both mycelial dry weight and cordycepin content of $P$. japonica is necessary. The highest cordycepin content was observed under the red-blue light conditions at $1654.8 \mu \mathrm{g} / \mathrm{mL}$, which was significantly different from that under the red-green $(397.5 \mathrm{mg} / \mathrm{mL})$ and greenblue light conditions $(74.8 \mathrm{mg} / \mathrm{mL})$.

Figs. 5-B and 5-C show the cordycepin content under different illumination times and that under a combination of different red-blue LEDs, respectively.

The highest cordycepin content was observed under the 12-h/day light condition and combination of three red and seven blue LEDs $(1657.5 \mu \mathrm{g} / \mathrm{mL})$. According to the results of the cordycepin content, the red and blue LED-wavelength combination was suggested to be appropriate for cordycepin production of $P$. japonica. We assumed that the cordycepin content of the $P$. japonica mycelium increased as the sugar content increased, and the cordycepin content was measured according to the sugar content of the medium. At this time, the light applied was under a 12-h/day illumination time and a combination of three red and seven blue LEDs. The results are shown in Fig. 5-D, and the cordycepin content of Cordyceps in a submerged cultured medium containing $7.5 \mathrm{~g}$ of sugar per $50 \mathrm{~mL}$ of medium was $1817.4 \mu \mathrm{g} / \mathrm{mL}$. From these results, we confirmed that the sugar content of the medium affected the cordycepin content of $P$. japonica. The results were used to derive the optimal conditions for maximum cordycepin content from $P$. japonica using RSM.

\subsection{Optimization of cordycepin production by RSM (BBD)}

The complexities and uncertainties associated with mushroom mycelial cultivation were reported to usually arise from lack of knowledge regarding the sophisticated interactions among various factors. Our preliminary data indicated that several major variables affected the performance of the culture in terms of cordycepin production. These variables are LED illumination time, sugar content in the medium, and incubation time in the shake flask (Table 1). The matrix corresponding to BBD is shown in Fig. 6 together with the observed experimental data.

Second-order model equation [Eq. (2)]:

$$
\begin{aligned}
\mathrm{Y}_{1}= & 3224.64+974.20 \mathrm{X}_{1}+571.52 \mathrm{X}_{2}-307.48 \mathrm{X}_{3} \\
& +250.54 \mathrm{X}_{1} \mathrm{X}_{2}-187.12 \mathrm{X}_{1} \mathrm{X}_{3}-95.55 \mathrm{X}_{2} \mathrm{X}_{3} \\
& -1651.51 \mathrm{X}_{1}^{2}-272.72 \mathrm{X}_{2}^{2}-663.51 \mathrm{X}_{3}^{2}
\end{aligned}
$$

This model fit was investigated using coefficient of determination $R^{2}$, which was calculated to be 0.984 , indicating that $98.4 \%$ of the variability in the response could be explained by the model. The $F$ values of the overall regression were significant at the upper 5\% level, which further verified that the second-order model is adequate for approximating the response surface of the experimental design. After transforming Eq. (2) to its canonical form, the optimum combination for cordycepin content $\left(\mathrm{Y}_{1}\right)$ was found to be the following: illumination time $\left(\mathrm{X}_{1}\right)=17.7 \mathrm{~h} /$ day, sugar content in the medium $\left(\mathrm{X}_{2}\right)=9.7 \mathrm{~g} / 50 \mathrm{~mL}$, and incubation time $\left(\mathrm{X}_{3}\right)=61.2 \mathrm{~h}$ (Fig. 6).

The model predicted a maximum response of $3779.2 \mu \mathrm{g} / \mathrm{mL}$ cordycepin yield. Verification of the calculated maximum value was done by the experiments that were performed in the culture media representing the obtained optimum combination, and a cordycepin yield of $3691.9 \mu \mathrm{g} / \mathrm{mL}$ (average of three 

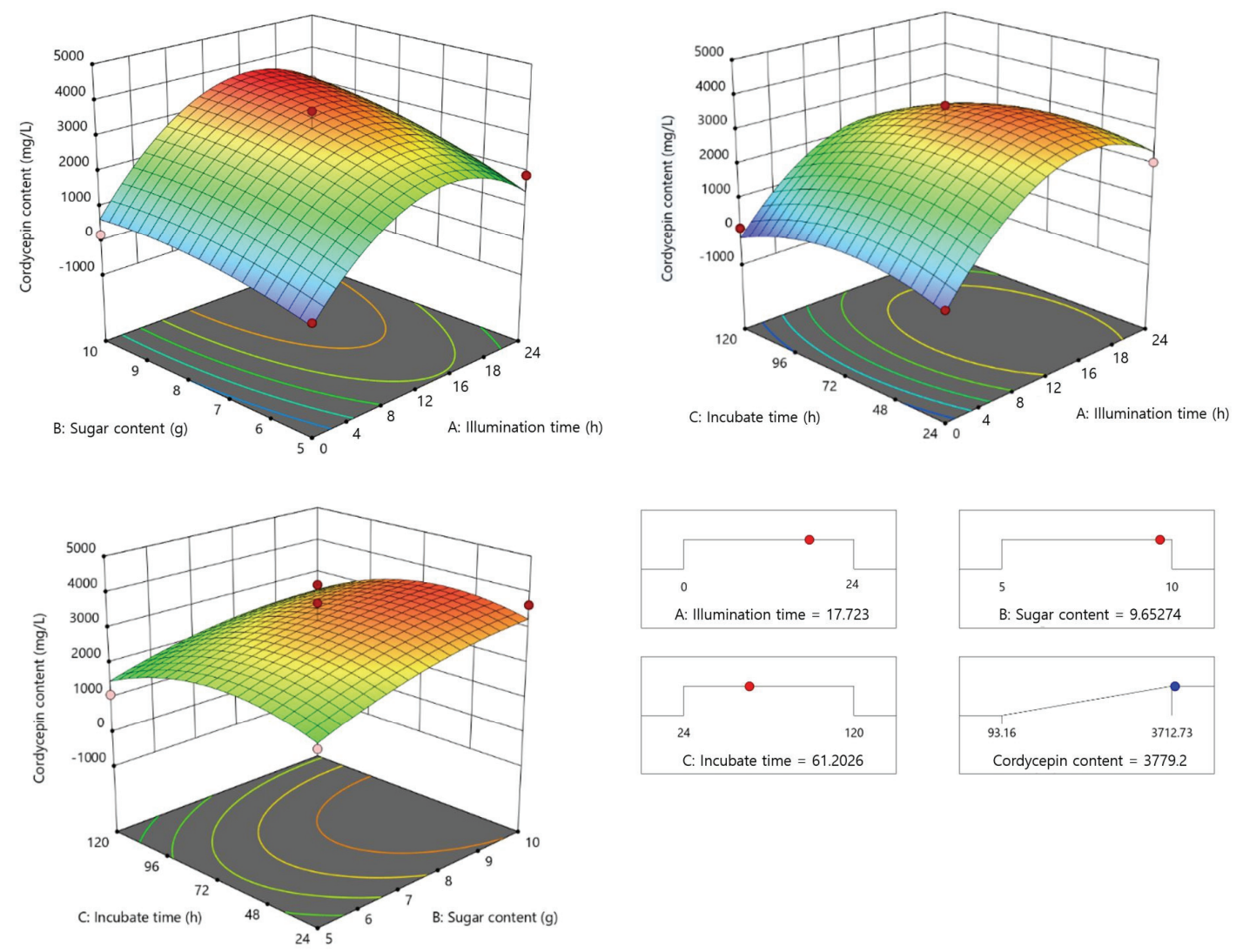

Fig. 6. Regression analysis of the Box-Behnken design experiments.

repetitions) was obtained. Although the measured value did not perfectly agree with the value predicted by the response model, it was similar to the latter.

Submerged cultivation of Cordyceps is seen as a promising alternative to chemical synthesis and solid cultivation for cordycepin production. Our research also proved that $P$. japonica contains high amounts of cordycepin. The maximum production $(3691.9 \mu \mathrm{g} / \mathrm{mL})$ of cordycepin obtained in the present study was significantly higher than that previously reported (Mao and Zhong, 2004; Mao et al., 2005). The highest value of mycelial dry weight of $P$. japonica produced in submerged culture was realized when the cultures were illuminated by green LED (Fig. 3). This result was similar to that of the study by Dong et al. (2013), which indicated that green light enhanced the mycelial growth of $P$. japonica when grown in a submerged culture. However, we found that blue light reduced the mycelium growth, which suggested that long LED wavelengths are beneficial for mycelial growth of $P$. japonica. In the present study, the cordycepin contents $(2412.5 \mu \mathrm{g} / \mathrm{mL})$ were higher than those reported by Liang et al. (2014) $(1.78 \mu \mathrm{g} / \mathrm{mL}$ of cordycepin content of $P$. japonica) when grown under the same light conditions (Fig. 3).

In the present study, the highest cordycepin content in $P$. japonica was under the red-blue LED combination conditions (Fig. 5), which indicates that red-blue 
LED combinations could enhance the cordycepin biosynthesis in P. japonica. The cordycepin content was generally better when the cultures were illuminated by the combinations of two LED wavelengths compared with those using only a single LED wavelength. A red-to-blue ratio of 3:7 was demonstrated to have the greatest effect on the cordycepin content, which was significantly higher than that of single-light illumination.

Dong et al. (2013) revealed that pink light (redblue) enhanced the cordycepin content in the fruiting bodies of Cordyceps. In the current study also, the cordycepin content in P. japonica under a red and blue light combination was significantly higher than that of cultures grown under other LED combinations (Fig. 5). Overall, according to the results of the cordycepin content, the combinations of LED wavelengths were more beneficial than single LED sources for high cordycepin content of $P$. japonica in submerged culture.

\section{ACKNOWLEDGMENT}

This study was carried out with the support of 'R\&D Program for Forest Science Technology (Project No. 2020252B10-2021-0001)' provided by Korea Forest Service (Korea Forestry Promotion Institute).

\section{REFERENCES}

Ahn, Y.J., Park, S.J., Lee, S.G., Shin, S.C., Choi, D.H. 2000. Cordycepin: Selective growth inhibitor derived from liquid culture of Cordyceps militaris against Clostridium spp. Journal of Agricultural and Food Chemistry 48(7): 2744-2748.

Baik, J.S., Kwon, H.Y., Kim, K.S., Jeong, Y.K., Cho, Y.S., Lee, Y.C. 2012. Cordycepin induces apoptosis in human neuroblastoma SK-N-BE(2)-C and melanoma SK-MEL-2 cells. Indian Journal of Biochemistry and Biophysics 49(2): 86-91.
Blumenstein, A., Vienken, K., Tasler, R., Purschwitz, J., Veith, D., Frankenberg-Dinkel, N., Fischer, R. 2005. The Aspergillus nidulans phytochrome FphA represses sexual development in red light. Current Biology 15(20): 1833-1838.

Chen, H.B., Wu, J.Y., Wang, C.F., Fu, C.C., Shieh, C.J., Chen, C.I., Wang, C.Y., Liu, Y.C. 2010. Modeling on chlorophyll a and phycocyanin production by Spirulina platensisunder various light-emitting diodes. Biochemical Engineering Journal 53(1): 52-56.

Cheng, C.W., Chen, C.K., Chang, C.J., Chen, L.Y. 2012. Effect of colour LEDs on mycelia growth of Aspergillus ficuum and phytase production in photo-fermentations. Journal of Photochemistry and Photobiology B: Biology 106: 81-86.

Chiang, S.S., Liang, Z.C., Wang, Y.C., Liang, C.H. 2017. Effect of light-emitting diodes on the production of cordycepin, mannitol and adenosine in solid-state fermented rice by Cordyceps militaris. Journal of Food Composition and Analysis 60: 51-56.

Cui, J.D., Zhang, B.Z. 2011. Comparison of culture methods on exopolysaccharide production in the submerged culture of Cordyceps militaris and process optimization. Letters in applied microbiology 52(2): 123-128.

Cui, J.D., Zhang, Y.N. 2012. Evaluation of metal ions and surfactants effect on cell growth and exopolysaccharide production in two-stage submerged culture of Cordyceps militaris. Applied biochemistry and biotechnology 168(6): 1394-1404.

Danesi, E.D.G., Rangel-Yagui, C.O., Carvalho, J.C.M., Sato, S. 2004. Effect of reducing the light intensity on the growth and production of chlorophyll by Spirulinaplatensis. Biomass and Bioenergy 26(4): 329-335.

Dong, C.H., Xie, X.Q., Wang, X.L., Zhan, Y., Yao, Y.J. 2009. Application of Box-Behnken design in 
optimisation for polysaccharides extraction from cultured mycelium of Cordyceps sinensis. Food and bioproducts processing 87(2): 139-144.

Dong, J.Z., Lei, C., Zheng, X.J., Ai, X.R., Wang, Y., Wang, Q. 2012. Light wavelengths regulate growth and active components of Cordyceps militaris fruit bodies. Journal of Food Biochemistry 37(5): 578-584.

Dong, J.Z., Ding, J., Yu, P.Z., Lei, C., Zheng, X.J., Wang, Y. 2013. Composition and distribution of the main active components in selenium-enriched fruit bodies of Cordyceps militaris link. Food chemistry 137(1-4): 164-167.

Guo, P., Kai, Q., Gao, J. 2010. Cordycepin prevents hyperlipidemia in hamsters fed a high-fat diet via activation of AMP-activated protein kinase. Journal of Pharmacological Sciences 113(4): 395-403.

Ha, S.Y., Jung, J.Y., Kang, H.Y., Kim, T.H., Yang, J.K. 2020. Tyrosinase activity and melanogenic effects of Rhododendron schlippenbachii extract In vivo and In vitro. Journal of the Korean Wood Science and Technology 48(2): 166-180.

Hidayat, A., Turjaman, M., Faulina, S.A., Ridwan, F., Irawadi, T.T., Iswanto, A.H. 2019. Antioxidant and antifungal activity of endophytic fungi associated with agarwood trees. Journal of the Korean Wood Science and Technology 47(4): 459-471.

Hsu, T.H., Shiao, L.H., Hsieh, C., Chang, D.M. 2002. A comparison of the chemical composition and bioactive ingredients of the Chinese medicinal mushroom Dong Chong Xia Cao, its counterfeit and mimic, and fermented mycelium of Cordyceps sinensis. Food Chemistry 78(4): 463-469.

Imtiaj, A., Lee, T.S. 2007. Screening of antibacterial and antifungal activities from Korean wild mushrooms. World Journal of Agricultural Sciences 3(3): 316-321.

Kim, H.G., Shrestha, B., Lim, S.Y., Yoon, D.H., Chang, W.C., Shin, D.J., Han, S.K., Park, S. M., Park, J.H.,
Park, H.I., Sung, J.M., Jang, Y., Chung, N., Hwang, K.C., Kim, T.W. 2006. Cordycepin inhibits lipopolysaccharide-induced inflammation by the suppression of NF-kappaB through Akt and p38 inhibition in RAW 264.7 macrophage cells. European Journal of Pharmacology 545(2-3): 192-199.

Kim, S.W., Hwang, H.J., Xu, C.P., Sung, J.M., Choi, J.W., Yum, J.W. 2003. Optimization of submerged culture process for the production of biomass and exo-polysaccharides by Cordyceps militaris C738. Journal of Applied Microbiology 94(1): 120-126.

Kodama, E.N., McCaffrey, R.P., Yusa, K., Mitsuya, H. 2000. Antileukemic activity and mechanism of action of cordycepin against terminal deoxynucleotidyl transferase-positive $(\mathrm{Td}+)$ leukemic cells. Biochemical Pharmacology 59(3): 273-281.

Leung, P.H., Zhang, Q.X., Wu, J.Y. 2006. Mycelium cultivation, chemical composition and antitumour activity of a Tolypocladium sp. fungus isolated from wild Cordyceps sinensis. Journal of Applied Microbiology 101(2): 275-283.

Liang, Z.C., Liang, C.H., Wu, C.Y. 2014. Various grain substrates for the production of fruiting bodies and bioactive compounds of the medicinal caterpillar mushroom, Cordyceps militaris (Ascomycetes). International Journal of Medicinal Mushrooms 16(6): 569-578.

Lin, R., Liu, H., Wu, S., Pang, L., Jia, M., Fan, K., Jia, S., Jia, L. 2012. Production and in vitro antioxidant activity of exopolysaccharide by a mutant, Cordyceps militaris SU5-08. International Journal of Biological Macromolecules 51(1-2): 153-157.

Ling, J.Y., Sun, Y.J., Zhang, H., Zhang, C.K. 2002. Measurement of cordycepin and adenosine in stroma of Cordyceps sp. by capillary zone electrophoresis (CZE). Journal of Bioscience and Bioengineering 94(4): 371-374.

Liu, Z., Li, P., Zhao, D., Tang, H., Guo, J. 2011. 
Effect of Light-Emitting Diodes on Cordycepin Production in Submerged Culture of Paecilomyces japonica

Anti-inflammation effects of Cordyceps sinensis mycelium in focal cerebral ischemic injury rats. Inflammation 34(6): 639-644.

Mao, X.B., Zhong, J.J. 2004. Hyperproduction of cordycepin by two-stage dissolved oxygen control in submerged cultivation of medicinal mushroom Cordyceps militaris in bioreactors. Biotechnology Progress 20(5): 1408-1413.

Mao, X.B., Eksiwong, T., Chauvatcharin, S., Zhong, J.J. 2005. Optimization of carbon source and carbon / nitrogen ratio for cordycepin production by submerged cultivation of medicinal mushroom Cordyceps militaris. Process Biochemistry 40(5): 1667-1672.

Ng, T.B., Wang, H.X. 2005. Pharmacological actions of Cordyceps, a prized folk medicine. Journal of Pharmacy and Pharmacology 57(12): 1509-1519.

Shih, IL., Tsai, KL., Hsieh, C. 2007. Effects of culture conditions on the mycelial growth and bioactive metabolite production in submerged culture of Cordyceps militaris. Biochemical Engineering Journal 33(3): 193-201.

Souilem, F., Fernandes, A., Calhelha, R.C., Barreira,
J.C.M., Barros, L., Skhiri, F., Martins, A., Ferreira, I.C.F.R. 2017. Wild mushroom and their mycelia as sources of bioactive compounds: Antioxidant, anti-inflammatory and cytotoxic properties. Food Chemistry 230: 40-48.

Tian, X., Li, Y., Shen, Y., Li, Q., Wang, Q., Feng, L. 2015. Apoptosis and inhibition of proliferation of cancer cells induced by cordycepin. Oncology Letters 10(2): 595-599.

Tuli, H.S., Sandhu, S.S., Sharma, A.K. 2014. Pharmacological and therapeutic potential of Cordyceps with special reference to Cordycepin. 3 Biotech 4(1): 1-12.

Tuli, H.S., Sharma, A.K., Sandhu, S.S., Kashyap, D. 2013. Cordycepin: a bioactive metabolite with therapeutic potential. Life Sciences 93(23): 863869.

Wu, J.Y., Chen, H.B., Chen, M.J., Kan, S.C., Shieh, C.J., Liu, Y.C. 2013. Quantitative analysis of LED effects on edible mushroom Pleurotus eryngiiin solid and submerged cultures. Journal of Chemical Technology \& Biotechnology 88(10): 1841-1846. 